\title{
CDISC SDTM Control Type Terminology
}

National Cancer Institute

\section{Source}

National Cancer Institute. CDISC SDTM Control Type Terminology. NCI Thesaurus. Code C66785.

Terminology codelist used with Control Type within the Clinical Data Interchange Standards Consortium Study Data Tabulation Model. 